\title{
$[53 x]$
}

\author{
VI. \\ Das Spierelbarometar; \\ v 0 in \\ Dr. Romenemauser.
}

Unfere Barometerbeobachlungen würden nuftreitig fehr an Genanigkeit und Branchbarkeit gewinnen, wenn fich die vielen anf der Erdliche zerftrenten Be: obachter über den Gebrauch gleichförmigerer Infixumente vereinigen wollten. Wir find zwar in Allgemeinen darin einverfianden, dals das fogenanute Heber - oder Sohenkelbarometer allein mathematilche Genaugkeit gewihren kann, whinend alle noch lo künflich zufammengefelzton Gefaffsbarometer, wie alle mit Schwimmern verfohene Infrumente etc. mehr oder minder trüglich und unficher find. Diefe Unficherheit lenchtet zwar bilon ans theoretilohen Grinden ein, fie tritt aber an dontlichlen hervor, wenn man dergleichen Infirumente längere'Zeit hindurch, vorzüglich in den Extremen der Scale, mit einem guten Heberbarometer zu vergleichen Gelegenlieit hat; indem dje Berichtigung diefer Inftrumente liäniig nur auf eine einzige Vergleichung mit dem Heberbaroneter gegründet ift, ohne alle Rückficht auf die ferriern nuausbleibliclien $\Lambda$ bwoichungen in Gange. Es wärde daher weit vorzuglicher feyn, wenn ficin die vielen Beftrebungen zur Vervollkommnung diefes wichtigen phyfikalifchen Infirumenies, dem einfachen und al- 


\section{$[352]$}

lein richtigen, aber doch in molufacher lWinficht noch bedürtigen Heborbaromeier vidmen, und es danu znr allgememen Anwendung emplehen wolien. Wir gefallen uns indefs in neuerer Leit ganz vorzüglich in künflich verwickelten 'Zulammenfizungen, die doch, aufser der dadurch erlıöleten Koftbarkeit, an fich wenig Empfelinngswerthes haben. Zierliche Nonien und micrometrifche Vorrichtungen geben folchen Inftrumenten den Schein von willenlchaftlicher Genanigkeit, wâhrend fie oft die gröblen Unrichtigkeiten verdecken nnd ihre Auffurlung lelbli erflinweren.

Der neuerwachte Sinn für. Baroneterbeobachtnngen könnte gerade jetzt für die Erd - und Naturtunde gewil's Grofses leifien, wemn mehr richtige und dabei billige Infrumente verbreitet, dagegen aber alle fabrikualsig gefertigten fogenannten Weltergläfer ausgefchlollen würden; indellen, bis jetzt finden wir diel'elben logar noch hänfig den officiell eingereiclnen Beohachinngsliften zum Grunde liegend. Diele Ve: berzeugung und der Wunfch, zur Erreichung des obigen Zwecks einen, wenn auch nur geringen, Beitrag zu liefern, veranlafsten mich, dielen Gegenfian. de bereits feit längerer Zeit Fleil's und Aufmerklamkeit zn widmen, und crmuthigen mich, die Refultate diefes Befirebens hicr offentlich mitzutheilen. Dio Anfertigung des Spiegelbarometers ift einfach und loicht; ich rechnete daher bei der Verbreinung diefes verbefferten Heberbarometers vorzüglich auch anf die, als cine angenehme und nützliche Nebenbelchäfiging unter den Fremender Naturkunde gegenwïtig allgemeiner fich verbreitende, Glasblafokunft - und werde daher, wegen der für den weniger Geübten 


\section{[ 550 ]}

nohkwendigen Unfändlichkeit in der Darftellung bei dem Manne vom Vache Entfoluldigung findes.

Zur Rechtfertigung meines Unternehmens ill es indefien nothwendis, daf's ich zuror in Algeneinen die Hauptbedürfnille bezeichne, worauf ich gegenwärtig noch, nach den verdienfvollen Leiliungen fo vieler gelehrter Whfiker und Michaniker, eine Yer. vollkommnung des Heberbaromelers gründele. Sic find folgende:

1) Tin normales, erleichtertes und geficherteres Auskochen aler Barometerröhren, als erjie Bublingung eines jeden pergleichuaren baroneters. Fien mit dicfer gefahrvollen und belchwerlichen Arbeit vertrant ift und die Unbehülflichkeit und Unzweckmälsiglkeit der von der ältefien, bis anf die nenefte Koerneriche Anleitung zur Baroneterverferligung empfollenen und gebranchen Kohlenbecken-lorrichtung kennt, wer ihr vorzinglich, wie ich, friher. hin einen Theil feiner Gelundicit geopfert hat, wird es leicht cinlelicn, dafs diefes nothwendige vollikommne Auskochen bei den meifien, vorzïglicis minder koftbaren Barometern, entweder nur fehr mangelhaft oder gar nicht verrichtet wird. Jene KohlenbeckenVorrichtung hat aber, wie aburliaupt das Ariskocher. in Kohlenfenex, mehrfache Nachtheile; einmal if, hei der dabei fo unbequenen Behandhug der Hönre, das Zerfpringen derfelben of bei gröster Voricht nicht zu vermeiden, der Arbeiter ift deshalb angltich und übereilt fich, da er, in einem folchen falle, den durch das Kolnlenfeuer angenblicklich expandirten, vergiftenden Quecklilberdämplen gar nicht entgelien kann; Codann ilt die, bei dieler Methodo nothwen- 


\section{[ 334 ]}

digo gegen den Horizont geneigte Lage der Rölsre gar nicht wohl geejgnet, Luft tund Waflerdünlte entweichen zu laften, indem beide am leichteften fenkrecht in die Höhe fteigen - und endlich kamn die Rölıro felbft nicht gehörig erfchüttert werden, um diele Entweichung zu erleichtern. Es mulste dalier zur allgemeinern Verbefferung der Barometer vor allen Dingen ein folcher Kochapparat gegeben werden, welcher völlige Sicherheit mit $Z$ weckmälsigkeit und Bequemlichkeit verbindet, und den jeder Barometer - Verfertiger alsdamn gewils gern anwenden wird, um leinen Infrumenten das erfte Erfordernifs zur Brauchbarkeit zu ertheilen.

2) Eine genauere, einfachere und bequemere Niveau-Beftimmung. Sie ift eins der loch wierighten Erfordernille und hat immer noch mehrere, fchwer zu beleitigende Mängel. Einmal erfordert fie eine vollkommen gleichweite Röhre, eine Bedingung, deren frrenge Erfüllung, bei der Länge der Rähre und der aus der bekannten Fabrikationsweife derfelben hervorgehenden conifchen Form, fehr fichwer zu erlangen ift. Man erleichterte fich zwar in nenerer Zeit dieles Erfordemifs dadurch, dals man den kleinen Schenkel von den obern Theil der Röhre abbrach, wo diefelbe die Scale des langen Schenkels erhieli, indent man beide Theile alsdann unten durch eine Faffung vereinigte. Indeffen habe ich fellft felur theure, mit den künftlichıften Micrometer - Vorrichtungen etc. verfehene Inftrumente diefer Art zu prüien Gelegenheit gehabt, welchen diefe Hanptbedingming to ganz fehlte, dals die Abweichung vom wahren Quecklilberftande an wehrem Stellen der Soale, eino Driferenz. 


\section{[ 335$]$}

von $\frac{x}{2}$ Linie erzengte. Wozu hier nun diefe kofibare, fich felbif lächerlich machende micrometrifche Vorrichtung? - Ueberhaupt halte ich diele Vorrichtungen, fo wichtig fie anch bei andern Inftrumenten find, doch gerade bei dem Barometer fiir völlig rïberflüfrig; es liegt in der TJatur der Sache, in dor Adhäfion und unficher begränzten Flïche des Queckfilbers, dal's fich bei diefem Inftrmmente die wirkliche Genauigkeit feimor Melfung nurr bis höchftens zul $\frac{\frac{1}{2}^{2}}{2}$ linie erftrecht, und diefe Theilung lälst fich mit gua ten Augen nnd bei einer zweckmäisigen Scale, etwa mit Beilülfe einer Loupe, weit leichlor und ficherer unmittelbar ablefen, als vermittelft eines folchen micrometrilchen Apparates *).

Sodann jft bei der zeitherigen Limriclitung die Beobachtung felbit za mülhfan und belchwerlich, als dafs dadurch die Genauigkeit nicht leiden follte. Ift die Röhre feftftehend und find beide Schenkel mit Scalen verfehen, fo werden die unvermeidlichen Fehler des Ablefens leicht verdoppelt und die Beobachtung wird zu umftändlich. Ift hingegen die liöhre, nack der Einrichtung von \$Luz, veríchiebbar, fo ift zwar dia Beobachtung erleichtert, allein das Inftrument

) Diefe allgetneine Bemerkung bezieht fich indeffen keineswegs suf gute, von gefchickten Mechanikern mit Fleifs und Keuntnils gearbeitete Inftumente (ihrer find nur wenige), fondern auf das jetzt feht moderne, lïgenhafte Micrometeruefen, wordurch jeder mechanifche Handwerker, ohne alle wiffenfchaftliehe Keuntnirs, in Geprãnge faubę polirter und gravirter Scalen und allerlei Schrauben-Vorrichtungen efc. dio fcliwere Kunft wirklicher Mechauiker aachaffen und fich ihnen gleichGellen will. 


\section{$[2301$}

lelbft verliert dadurch an leltigkeit und Daner. Ueberhaupt erlolien mir es aber immer als eine wefentliche Unvollkommenheit, dafs man, zur Berichtigurg der Horizontalebne, das Infrmment im Zimmer lo hoch auflı̈̈ngen muls, dals man bei jedor Beobachtung in die Hölıe zu fieigen genölhigt ifi, wn richtig ablefèn zu können.

5) Ift cs eine fichere Jirfahmong, da/s die oft unerkärliche Abweichung jelby der bogen, mit allem Fleifs erbaueten Baromeier, voriolich in den Extremen der Scale, in der Ferfiridenheit und Ver. änderlichkeil der torricellifillen Leere begriondet ift. Sey es enwa weniger Inft, Dünta, oder einc andere nocli mbekante Bateric, welch, den wenghens nicht ganz leer denkbaren liaun exfillen, und deren grölsere oder geringere Ansdelnung einen Einflul's auf die Maltung der Queckfiberfänle änfsern dieler Linfuls if nach meinen forglitigen Verfuchen venigfens uniangbar, und es ifí zur DarfelIkung guter und vollkommen correfpondirender Barometer nothwendig, $\dot{\mathrm{d}}$ afs dieler haun flets gleichbleilend und nomal angeordnot wird. Diefe Anordnnng verhindert zugleich bei minder lablewen binometem die Diferenz in Gange, wonn auch njolst in stande der Queckfilberfïule. Zia dem Inde nutis das Niveau oben an dom langen, und die Scale am knizen Schenkel angebracht werden. Durch diele pinrichtung wird die Bedingung der Gleichfömiglseit logleich erfüllt, es fpricht dariur aber auch folgendes: is ift nämlich weit naturgemülser, dals wir bet unlern Beobachtnngen die nunittelbare Wirkung des Luitdruckes bezeichnen, dieló erfolgt aber ant den kur- 


\section{[ 337 ]}

zen Sichenkel des larometers. Der fich fteigernde Druck treibt daher das Qnecklilber, dem Begriffe der Schwere zufolge, tiefer hirnab und nicht höher hinauf -; die Bewegung im langen Schenkel ift nur cine fecundiare Wirkung der Atmolphäre. Fs ift daher eine ganz uinallende Sprache, welche unfere neuern Bleberbarometer von den frühern Gefäs-Barometern übernommen liaben, welche freilich nur dis miltelbare Wirkung des Luftdrucks anlchaulich machien: fie mufs dem Unkundigen ganz unverfiündlich feyn, da fie bei Anbringung der Scale am kurzen Schenkei dem Angenfchein völlig widerfiricht. $O b$ nun gleich diele Bezeichnnngsweife in der Sache nichts andert utnd dem damit Vertrauten völlig gleichgültig leyn kann, lo würde doch aus den angefilhrtein Gründen, eine Unwandling des Ansdrucks fehr empfehlenswerilh feyn.

4) Gröfsere Dauer und Sicherheit bei dem Transport des Barometers. Es giebt viele, felnr künftich erbancle Inftruncute, die man kaun ohne Gefaln der Zerförung in Zinnmer von einem Orte zum andern tragen kamn; manche davon liaben die noch üblere Eigenfcliaft, dafs fie bei geringer Erl'clıütterung leicht unnerklich jetwas Luft fchöpfen und den Beobachter dadurch oft anf längere Zeit betrügen. Lin jedes Barometer follte wenigftens fo eingerichtet feyn, dafs es ohne Nachtheil herumgetragen werden kann, da ja oft nur die örtlich verfchiedenc, mit demfelben Inftrumente gemachie, Beobachtung die vollkonmenfte Genauigkeit gewïluren kamm. Diefe Bedingung lälst fich aber am beften dadurch erfüllen, dafs das Barometer eine folche Einrichtung ex- 


\section{[ 338$]$}

halt, vermittelft welcher der Qnechlinerlule ein, alles Schwanken rerhintencies, Gegengewicht gegehen wird, welches zugleich die zerfö̈renden Wirkungen der abwechfelnden Ausdelnung durch Wärme nud Kälte befeitigt.

Diefes waren ldie anf längere Erfilhrung gegrïndeten Anfichten, wclche mich bei meinen Verluchen zur Verbeflerung des Harometers leiteten. Da ich Einfachheit und Daner bei allen meinen Mafchinen ganz vorzüglich zn erftrcben fuche, fo hofle ich, dals fie anch das Spiegelbarometer vortheilhaft auszeichnen werden. Ich erreichte diefes vorzüglich durch Anwendung eines kleinen, felur fichern Glas -Stiefels, welcher das, durch die Verinderung der Aimofphäre wechfelnde, Maafs des Queckfilbers vermittelft feines Kolbens anfnimmt oder erfetzt. Frühere Verfertiger ähnlipcher Vori:ichtungen bedienten fich eines Lederbeutels etc., welcher indefen durch foine nochlallende Ausdehnung fowohl die Bewegung als anch den feften Stand der Queckfilberfäule unfiches macht, durch Preffing leicht Ausfch witzungen erleidet und fo oft die koftbarie Vorrichtung bald ganz unbranchbar macht. Nach dirlen Benterkungen wende ich mich nun zar Darfielhng der Vorrichtnngen lelbft.

Der Apparat zum Auskochen der Barometer-Röhren. Fig. I.

Irgendwo an einer WVand wird in pantender Höhe die elaftifche Holslattep ficher befefigt und am andern Endo derfelben a der eiferne linken $r$ anfgefchroben. Man nmwindet alsdann diefen Haken mit einer wollenen Sshmur, damit die Glasröhre ohne Befchädigung daranf ruhen kann. 


\section{$[539]$}

$t$ ift eine Syirituslampe, welche vermiltelf der Röhre $m$ in der Mitte lo durchbrochen ift, dals die Blasröhre $s$ vollkommen gerärmig hindurch gefteckt werden kann; das Innere diefer R.öhre ift mit einem folıwachen Stück Filz bis nahe zum oberen Pande ausgefüttert. Rings um diefe Röhre $m$ bilden 4 Dochte eine fiarke Tlanme nm die durchgefteckte Barometer-Röhre, welche vermittelif des Handgriffs der laampe bequem an derfelben anf und nieder bewegt worden kann. Fig. $x$ zcigt den Dochthalter dieler Lampe von oben, $m$ ift die geränmige, nuittlere Oeffnung, welchic die Barometer-hïhre anfnimmt und $d d d d$ find die Oefnungen für die 4 Dochte. $W$ if ein lenkrecht unter der Vorrichtung ftehendes, geränmiges $t$ alfergefojs.

Das Anskochen vermiltelft dieles $A$ pparates ift frhr leicht und bequem, es ift hier an einer gewöhnlichen Heberbarometer-Rölıre dargeftellt. Iie forgfältig gefüllte Rölire os wird an den Haken $r$ aufgehangon und mit einem Faden dafelbft feftgebunden. An den kurzen Schenkel o befefigt man eine leichte $\mathrm{Pa}$ pier-Rolle, welche man leitwäts in ein nebenftehendes Glas mit Viafer leitet, um die etwanigen Dämpfe, wie das etwa überfteigende Queckfilber dafelbft aufzufangen. Man zündet alsdann die Lampe $l$ an, läfst die Cenkrecht herabhängende Rölire $s$ durch die Oeffnung $m$ hindurchgleiten und erhitzt anfangs durch Cchnelles Heranf-und Hinabbewegen der Lampe die ganze Röhre allnuälig und gleich förmig. Alsdann füngt man unten bei $s$ das eigentliche Kochen an und l'chreitet damit nach nnd nach an der Röhre hinauf. Durch Bewegung der Lampe ober - und unterhalb des 


\section{$[5 i 9]$}

Kochymultes kann man fehr leicht das fohnelle und der Röhro gefihrliche linrabfallen des fieigenden Queckfilbers verhülen, wozn f́chon der erfie Verfich linlängliche Anleitųng giebt. Während diefier Operation giebt cin Gehülfe fortwährend nit einem Hammer $c$ kleine Schlüge auf die Holzlatte, welche dadurch in andauernde Vibration gefelzt wird und das fenkrechte Auffeigen der Lanft und Waflerdämple in der Röhre ungemein befchlemigt und fördert. Die Vibration der Holzlatte kann anch ohne Gelü̈lfen durch einen einfachen Fufstritt leicht bewirkt werden.

Das Auskuchen der Rölire erfolgt anf diefe Weife fo fohnell whit vollkommen, als es anf keinem andern $\mathrm{W}$ ege möglich iff; nan ifi dabci lo ganz gegen alle feluädlichen Ausdünftungen des Queckilibers we auch gegen jeden Verhuft diel'es Metalls, gefichert, dafs felbfi in dem kamm möglichen Falle des 'terfpringens anch nicht der kleinfte Theil des Geckiflbers zerfirent werden kam, donn der ganze linhalt dor Höhre fält augenblicklich in das Wallergefats $t r$ herab. Joh darf daler hofen, dal's diefe lehr wento helsbare Vorrichtung zum Befien der Baroneser wad it.rer Vorfertiger cinen allgemeineren Luidul linden wird.

\section{Das Splegeibaroneter. Fig. 2.}

$e$ ift der lange und $b$ der kurze Schentel dieles Barometers, $i$ das die Quncksiber - Temperatur anzejgende Themometer, und $a$ ein kleiner; nit einen Kolben verlehener Stiefel von Glas. Alle dide Bübren find durch ein Parallelcpipion von cintuia recht dichten and harten Holze, $M$ in Verbindung geletzt. 


\section{$\left[\begin{array}{lll}341 & 3\end{array}\right.$}

Zu dem Ende wird letzteres mit folgenden Bohröffnungen verfehen :

1) Die horizontale bis zu $g$ lindurchlaufende Oeffnung $c$, welche die Communication der Röhren etc. bezweckt.

2) Die fenkrecht anf vorige treffenden Oeffnungen, $m$ für den kurzen Schenkel, $p$ für den langen Schenkel und die weitere $g$ für das in das Queckfilber verfenkte Thermometer.

3) Die Oeffnung a für den Glas-Stiefel, welcher zugleich als Queckfilber - Behälter dient.

Man bohrt diefe Oeffnungen am beften auf der Drehbank vermittelft eines fogenannten, den Durchmeffer der Röhren um etwas über fícigenden CentuumBohrers, wodurch fie, fowohl recht glatt, als vollkommen aufeinander treffend erhalten werden. Das Innere diefer Höhlungen tränkt und überziehet man alsdann mit einer ftarken, klaren Fifchlein-Anflöfung, wodurch diefelben fowohl an Dichtigkeit, als an glasartiger Glätte gewinnen.

Zu dem Stiefel $a$ wählt man ein, etwa/4 bis 5 Linien im Durchme fer haltendes und $2 \frac{1}{2}$ Zoll langes vollkommen cylindrifches Stückchen einer ftarken Glasröhre, deren Ränder man fauber abfchleift und unterhalb etwas weniges an der Lampe erweitert und glättet. Am aufsern Umfange wird das Glas hingegen mit einer fcharfen Feile geritzt und rauh gemacht und mit einigen Lagen feinen Papiers oder dünner Kalbsblafe, vermittelft guten Fifchleims dicht überzogen. Wann diefer Ueberzug völlig trocken ift, lo bearbeitet man ihn mit der Feile fo weit, dafs die Röhre genan fchliefsend in ihre Holzöffunng einpafst, wofelbft fio alsAnnal. d, Ptyfik. B. 8m. St. 3. J. 1825 . St. 7. 


\section{[ 3421}

dann mit einem nenen Leimantrich Porgfültig eingefïgt wird.

Der für diefen Stiefel beftimmte Kolben a befteht aus einem Stückchen recht feinem, dichtem und elaftifchem Kork. Es wird durchbohrt und zwifchen 2wei an dem Schraubenftift $d$ angebrachten eifernen Scheibchen bei $a$ feft aufgefchraubt, mit einem MeLfer ziemlich rundizugefchinitten und auf der Drehbank völlig paflend abgedrehet. Man erlangt dieles am leichteften durch ein recht fcharfes Rafirnefíer, indem man die Schneide deflelben faft fenkrecht auf die Drehungsfläche anfetzt. Auf diele W Weile erhält man den Kolben vollkommen glatt und rund; die Anwendung der Feile nunfs hingegen vermieden werden, da fie den Umfang fafserig und nie ganz glatt darftellen kann. Der Kolben mufs fich in der Rölhre etwas preffen und vollkommen dicht f'chliefsen; da die Bewegung deflelben vermittelft einer Scliraubenvorrichtung bewirkt wird, fo lat fie auch bei dem dichteften Schlufs keine Schwierigkeit. Die Kolbenflange ift unterhalb des Kolbens, fo weit als die Linie ilhrer Bewegung reicht, vierkantig gearbeitet und läuft dafelbft in der gleichförmigen. Oeffnung des an dem unten vorftehenden Brette befeftigten Melfingftiucks $r$, welches zugleich den Stiefel verfchlielst, und bewirkt, dafs fich der Kolben nicht drehen, fondern nur auf und nieder fteigen kann; weiter nach unten bildetalsdann die Kolbenftange eine "Schranbe, welche durch die unter dem Brette frei hervorragende Fingerfchranbe bewegt wird, indem diefe fich in cinem Falze drehet und fo den Kolben auf und nieder ziehet. Alle mit dem Queckfilber in Berührung kommende Metall- 


\section{$\left[\begin{array}{lll}343 & 1\end{array}\right.$}

theile müfen von Eifen oder Stahl gefertigt werden; fie werden gut polirt nnd gegen das Roften mit dem Anftrich eines harten Bernfteinfirnilles verfehen.

Ift die Vorrichtung fo weit vorbereitet, fo wird das in Hinficht feiner Scale bereits vollkommen berichtigte Thermometer bei $g$ eingeletzt. Die Röhre wird zu dem Ende zunäclıft über der Kngel ganz wie der Glasfitiefel behandelt, mit einem Streifen Blafe verfehen und in einem Kork recht feft verleint. Der Kolk wird für die ihm beftimmte Oeffnung rund und paffend zugefchnitten, fo dafs fein Durchmefler den der Kngel um etwas ïberfchreitet, damit die Kugel ohne anzuftofsen allenthalben vom Queckfilber des Barometers umgeben ift. Mit diefer Vorficht letzt man nun den Kork mit Leim in feine Oeffnung ein und verfchliefst alsdann auch die Bohröffnung $c$ des Holzftücks $M$ entweder mit einer palfenden Holzfchranbe oder einem Kork, welche man einleimt.

Wir wenden uno nun zur Anfertigung der Barometer-Röhren $b$ und $e$. Man wällt beide etwa von einem innern Durchmefter von $1 \frac{\pi}{2}$ Linie. Es ift indeffen nicht unumgänglich nothwendig, dafs beide von völlig gleichem Durchmeffer find - wenn diefes Verhältnil's nur nicht auffallend abweicht.

Der lange Schenkel e $p$. Man nimmt dazu eine reine, wenn auch nicht gleichweite oder vollkommen cylindrifche Glasröhre von 35 Zoll Lünge. Sie wird an dem Ende $p$, wo lie in das Holz geleint werden foll, glatt gefchmolzen und wie die uibrigen Röhren mit Blafe überzogen, welche mit der Feilo für ihre Oeffnung vollkommen paftend genracht wird. Alsdann wird fie gut gereinigt und an Ende $e$ halbkugel- 


\section{[ 344 ]}

förmig zugefchmolzen; es ift wichtig, dafe diefer Verfchlul's nie konilch geformt fey, und dagegen vorzuziehen, dafs die Halbkugelform unmerklich über den Durchmefler der Röhre hervortritt. Die Röhre wird nun vermittelft eines Bindfadens am Kochapparat Fig. 1 bei raufgehüngt, erhitzt und mit reinem, gleiclıförmigg erhitztem und wohlgetrocknetem Queckfilber, durch einen feinen Papiertrichter gefüllt - und alsdann nach obiger Anleitung aufs fvollkommenfte ausgekocht.

Der kleine Schenkel $6 \mathrm{~m}$. Man wählt dazn ein recht klares und vollkommen cylindrifches Röhrftückchen, welches anch keine Schwierigkeit hat, da es nur etwa 8 Zoll lang zu feyn braucht. Es mul's zu dem Ende aufs forgfilligfte calibrirt werden. Es wird nun am untern Ende $m$ glatt gefchmolzen und oben bey $b$ mit einem etwas erweiterten Rande verfehen. Unten bey $m$ wird diele Röhre ganz wie die übrigen behandelt, mit Blale umllebt und reclit feft in die Bohröffnung eingeleinut.

Um nun auch den aurgekochten langen Schenkel bei $p$ einzuleimen, kehrt man $M$ fo herum, dafs $b$ und $t$ nach unten kommen und die Ocfnung $r$ des Stiefels nach oben fiehet. Das Inftrument bleibt in diefer Lage hängen, bis alles vollkonmmen trocken geworden ift, alsdann fchreitet man zur vollendeten Füllnng deffelben. Zu diefem $Z$ wecke fcliranbt nuan das Parallelepipedon $M$ an das bereits vorgerichtete Barometerbrett, wo daffelbe bis an die Röhren eingelafien ift und befeftigt die Röhren einftweilen nur oberflächlich nit einem Faden am Brette, um fie vor Zerbrechung zu fchützen. Man fchiebt jetzt in den kleinen Schenkel $b$ einen an einem Eifen- 


\section{[ 345 ]}

draht befeftigten Stöplè bis an feine Mündung $m$ hinauf, damit diele Röhre ganz verfchlofen ift. Nun giel'st man von oben durch den offnen Stiefel $a$ fo viel reines und trocknes Queck filber ein, dal's derfelbe etwa bis zu $\frac{2}{3}$ feiner Länge angefüllt ift, wobei man durch Erfchütterung und vermittelft eines Drahtes alle Luft aus der innern Höhlung auszutreiben fucht. Alsdann drückt man den Kolben nur lo tief in den Stiefel $a$, dafs derfelbe dadurch gelchlofen iff und neigt nun die Vorrikltung nach gerado zur Seite, fo dafs das Brett horizontal liegt und die fchmale Kante $n$ nach mnten gekehrt ift. Bei dicfer Lage fteigt dic zwifchen den Kolbem und Queckfilber im Stiefel eingefchlofene Luft in den Raum unter $c$ linauf, welches man durch einigo Erfchütterung leicht vollftändig bewirkt. Wenn man jetzt den Stöpfel aus dem kleinen Schenkel $b$ auszielıet, das Barometerbrett bei feinem Obertheil $e$ etwas hebt und zugleich den Kolben tiefer in den Stiefel einfchiebt, fo treibt das in $m b$ auffteigende Queckflber den unter $a$ befindlichen Reft der Iufft vor fich her. Hat das Qneckfilber anf diefe Weifo im kleinen Schenkel die Länge der Scale überfchritten, lo kann man das Barometer ohne Sorge aufluängen und die Füllung ift vollendet.

Man befeftigt num die Vorrichtungen $d$ und $r$ und die Röhren felbft mit gefütterten Meflunghüllen am Brette, anch fügt man fogleich die Barometer- und Thermometer-Scalen linzu. Die richtige Stellnng der letztern gefchiehet nach den zuvor an der Thermorneter-Höhre gemaclıten Merkzeichen, die Rectification der erftern aber wird anf folgende Art be- 


\section{[ $\begin{array}{ll}346 & \text { ] }\end{array}$}

wirkt. Hat man ein anderes gutes Heberbarometer zur Hand, fo fohraubt man den Kolben vermittelft der Fingerl'chraube $d$ f'ó weit herab, dafs das Queckfilber in dem kleinen Sclienkel $b$ auf der Scale einen gleichen Stand mit dem Heberbarometer angiebt. Bei diefem Verfahren wird nun anch die Queckfilberfänlo im langen Schenkel $e$ bis zu einem gewiffen Punkte w herabfinken, welches für die Folge der NormalPunkt des feftftehenden Niveau ift. Man bezeichnet denfelben einftweilen nur dadurch, dafs man hier ein, mit einem feinen Querftrich vorfehenes, Kartenpapier unter die Röhre fohiebt und läfst die Vorrichtung fo einige Tage ruhen, wälurend man den fernern Gang beobach tet und vergleicht; diefes erfordert, wegen einiger Senkung des. Queckfilber's etc. die folgende genaue Berichtigung des Nivean. Zn dem Ende trägt man auf eine mit dem langen Schenkel genau parallel aufwärts gezogene Linie, vernittelft eines fichern Zirkels, aus dem Theilpunkt $28^{\prime \prime}$ der Scale forgfältig 28 Paris. Zolle aufwärts und zieht durch den Endpunkt diefer Linie eine darauf fenkrechte quer über das Brett, welche nun die normale Lage für das $\mathrm{Ni}$ vean bezeichnet.

$S$ ift ein kleiner Metallfpiegel von der Höhe eines Zolles, welcher auf eine Melfingplatte verlöthet, und zur Seite der Röhre $e$, in einer Neigung von $45 \mathrm{Grad}$, gegen die Niveau-Linie und zwar lo feftgefchraubt wird, dafs feine Fläche fowohl fenkrecht auf dem Brette ftehet, als dal's anch die Niveau-Linie feine Mitte trift. An den Punkte, wo diele Linie den Spiegel berührt, wird alsdann auf der Spiegelfläche 


\section{[ 347 ]}

eine fehr feine Linio aufwärts und fenkrecht auf dem Brette ftehend eingerifen.

Das Nivean wn befteht ans einem Caubern Silberoder Elfenbein - Blättchen, auf deffen Mitte eine feine fchwarze Linie bezeichnet ift, dic fich, wie fchon bemerkt, an die Linie der Spiegelfläche genau! anfchliefst. Die Form diefes Niveau - Blättchens leuchtet ans der Zeichnung ein; es wird nach vollkommner Berichtigung mit der anf dem Brette bemerkten Linie nnverrückbar angefchraubt.

Das Barometer würde nun in feiner einfachften Geftalt vollendet feyn, wemn inan ilhm nicht noch einige pallende Verziernngen geben will; fo kann man z. B. dem unterhalb $M$ vorfiehenden Theil des Brettes bey $r$, welcher den Mechanismus der Kolbenbewegung enthält, in gleicher Höhe mit $M$ verdecken, wodurch zugleich das Eindringen von Staub in den Stiefel $a$ verhü̈tet wird, $\mathbf{u}$. . $w$.

Die Scale des Barometers hat aus oben angegebenen Gründen eine der gewöhnlichen entgegenge-

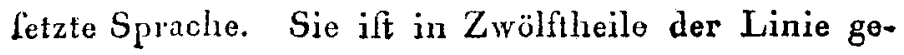
theilt, welche nan bei dem Gebranch einer Lonpe noch halbiren kamn*). Wer indelen andern künftlichen Vorrichtungen zu genauerer Maalsbeftimmung melir Vertrauen fchenkt, kanu diefelben leicht mit der

-) Ich verrichte diefe Theilung mit einer eigenthümlichen, fehr bequemen Theilmafchine - welche nicht alleis die Theilfriche in hoher Genauigkeit verzeichnet, fondern auch zugleich ihre, nach Anordnung der Scale verschiedene, Länge befinmt, fo dafs relbft ein Blinder diefe Theilungen machen kann. 


\section{[ 348 ]}

Scalo in Verbindung fetzen. Ich mefle ohno diefelben fchueller und ficherer, indem ich den Stand der Queckfilberfüulo nicht nach der obern VVölbung derfelben, fondern nach ihrer Bafis beftimme, welcho bei einer gut gewählten Röhre und richtiger Beleuchtung, durch einen fcharfen Schatten in derfelben, nicht allein den Horizontalftand des Auges berichtigt, fondern auch den genaueften Schnitt erlaubt.

Das zu beobachtende Spicgelbarometer wird l'o aufgehängt, dafs fich die Scale in der Augenhöhe des Beobachtenden befindet und die vordere Spiegelfläche dem Lichte zugekehrt ift. Bei der Beobachtung falst man die Schranbe $d$ und fiehet aufwärts in den Spiegel $\boldsymbol{S}$, wo man die Queckfilberfäule aufs fchürffte durch den reflectirten Niveau - Strich gefchnitten erblickt, indem das Bild derfelben in der Riclitung $x$ herab und zugleich in einem Winkel von $45 \mathrm{Grad}$ vor das Brett reflectirt wird. Man richtet dabei das Auge fo ein, dafs das reflectirte Niveau die zarto Linie im Spiegel deckt, wodurch jeder Fehler in der Vifirlinie unmöglich wird, Durch Bewegung der Schraube $d$ hebt oder fenkt fich nun die Queckfilberfänle, man kann fie daher, wegen der Länge der Richtungslinie auf einen Blick, aufs fchärffte und genanefte in das $\mathrm{Ni}$ veau einrichten. Nun liest man den Stand des Barometers auf der dem Ange gegenüberliegenden Scale ab. Die Leichtigkeit, Bequemlichkeit und Sicherheit diefer Beobachtungsweife wird fich gewils allgemeinern Beifall erwerben.

Die in Fig. 5 nach einem gröfseren Maafsftabe befonders dargeftellte Vorrichtnng fohützt endlich das Barometer bei dem Transporte gegen Belchädigung. 


\section{$\left[\begin{array}{lll}349 & 1\end{array}\right.$}

\section{$b$ ift ein Stïck-des kleinen Schentels bm (Fig.-9.)}

und ha eine gewöhnliche feine Thermoncter - Röhre von zureichender 'Glasftarke. : Bè $\cdot K$ wijgl 'eine fuatio Kugel angeblafèn, welcho obcrhalbmadi $a, z u$ in eine feirte 6 pitze ausgezogen wird. Dic nhytere Mündung $n$ wird etwas etweitert und auf belsannte VV cile mit Blafe überzogen und in ein Stückchen Kork cingeleimt, welches, wie die.Figs zeigt, in einen in dis Schenkelröhro genau paffenden Kolben verwandelt wird. Die untere etwas ansgehöhlı Flüche diel's kleinen Kolbens überzieht man mit einer Lage Fifcl.leim, um fie völlig glalt herzuftallen. Bei engern Rölren kann man fich auch anftatt des Korks eines ftarken elaftifchen Lederfïückchens bedienen.

Der Gebramch dieler Vorricht.ung ifi nun folgender: Wenn das Baroneter transportirt werden loll, fo fchraubt man den Kolben a (Fig. 2.) lo weit nach oben, dafs das Queckfilber im langen Schenkel oben bei e dicht anf'chliel'st und in kurzen über die Scale hinanffteigt; alsdann drückt man den kleincn Kolben $n$, wie Fig. 5 deutlich macht, dicht auf das Quecklilber herab; die zwifchen beiden befindliche luft entweicht dabei völlig durch die Röhre na und das Queckfilber fteigt bis in die Kngel $K$ hinauf. Auf diefe Veife ift nun, wegen des Gegendrucks dicfer Queckfilber - Säule in $n K$ das Barometcr vollkommen gegen das Eindringen von Luft gefichert. Dehnt die VWärne das Queckfilber des baroneters aus, fo fteigt es in die Kugel $K$ hinanf, zielıt es hingegen die Külte zufammen, fo finkt cs aus der Kugel nach. Das Schwanken der Queckfilberlä̈lle ift durch die Feinheit der Röhre verlindert. 


\section{[ 350 ]}

Nachdem das Barometer an Ort und Stelle anfgehăngt ift, zieht man dỉe kleine Kolbenrölıre wieder etwas in die Höhe, wobei der Luftdruck fämmtliches Queckfilber wieder in $b$ lierabtreibt und die Luft alsdann nach folgt. Jetzt f́chranbt man den langen Schenkel anf das Nivean und es kann nun die Bcobachtung gelchehen. Diele kleine Vorrichtung $n k i c$ bleibt zum Schutz gegen Staub etc. mit dem Barometer verbunden; fie wird zn dem Ende über $b$ (Fig. 2) in der Richtung der Linie $x$ mit belederten IVIClning-Hülen befeftigt, worin fie fich ftetig und ficher auf und nieder fchieben lälst *).

Wer das hier befchriebene Spiegel-Barometer ganz von Glas und olune Molzfaflung anfertigen will, findet in Fig. 3 und 4 einige zweckmäl'sige und leicht ausführbare Formen. Diele Einrichtung hat mehrere Vorzüge und ift jedem in der Glasblalekunft Geübten von felbft verftändlich. - **)

*) Aufser diefer, bei jeden folchen Barometer leicht anzubringenden, felur einfachen Vorrichtung habe ich auch oin eigenthümliches, fehr ficheres und bequemes Reifebarometer zufammengefetzt; es ift mit einem Hahn verfehen und der Wech. fel der Temperatur wird durch eine federnde Einrichtung des Kolbens a regulirt - indeffen wïrde die Befchreibung deffelben die Gränzen diefer Abhandlung überfchreiten, welche nur die einfachite Einrichtung des Spiegelbarometers zum Zweck hat. Ich behalte mir vor, diefe und melurere andere vortheil. hafte Barometer - Conftructionen etc. kün'tig vollfändijer in einer befonderu Schrift darzuftellen. $R$.

**) Eine Hauptabfioht des Hrn. Verfafers bei Bearboitung diefes Auffatzes war, Freunden der Phyfik, die in grofser Entfernung von roliden mechanifchen Werkfätten wohnen, und dagegen folbft im Befitz des unentbehrlickiten Goräthes find, An. 
leitung zu gejęn, fich ohne weitere Hulfe, ein Inftument za verfertigen, Jas wenig koftet, die gewöhnlichen Wettergläfer an Genauigkeit übertrifft und dabei noch einige fonftige Vortheile vereint. Dafs dem Vertaffer diefes gelungen, wird man nicht abftreiten wollen. Schwerlich aber möclten die hier vorgefchriebenen Einrichtungen den Auforderungen entrprechen, die man gegenwärtig in Betreft der Genauigheit an Infrumente diefer Art zu machen gewolnnt ift, und in der That von den beffern mechanifchen Krinftern bisher fchon befriedigt worden find. Die Genauigkeit von $\frac{x}{2 x}$ Linie, welche der Verfaffer fich als Gränze gefetzt hat, if, wenn gleich zu den meiften $Z$ wecken hinreichend, doch gewifs eine fehr matsige und bei einiger Sorgfalt in der Ausführung, mit Nonius und ausgefpannten Fäden, fchon ołne Loupe, leicht zu erreicheu. $O b$ indefs bei den vom Hrn. Verfaffer gewăhlten Vorrichtungen die innere Sicherheit der Meffung bis zu jener Gränze reicht, möchte ich rehr bezweifeln. Dio Art der Beftimnung des oberen feften Punktes, die Einrichtung der Scale, die hier zugleich aus Holz und Metall befteht, und der Umftand, dafs auf diefes Barometer nur der Stand eines anderen, als richtig angenommenen, übertragen wird, wobei wegen Temperaturverfchiedenheiten und anderer Zufalligkeiten leicht Irthümer entftehen können, find Dinge, die diefem Inftrument keine fehr grofse Präcifion geftatien, und ihm wenigftens kelnen Vorzug vor den fchon üblichen gewähren. Doch will icb damit nicht läugnen, dafs innerbalb gewiffer Gränzen diefes Barometer feine $Z$ wecke wohl erfüllen mag; auch in gewirs für nicht forgfältig ausgekochte Barometer die Einrichtung, das Vacuum auf conftantem Volumen zu erhalten, ganz zweckmärsig; nur müfste diefes Volumen, wenn man wirklich Gafe von merklicher Expanfivkraft darin vorhanden anzunehmen hat, bei einer forgfältigeren Berück fichtigung noch für die Temperatur eine Correction erleiden. Es wird indefs in jedem Fall ficherer feyn, auf das Auskuchen der Röhre die nöthige Sorgfalt zu ver* wenden, und bei der Beobachtung das Inftrument keine ungewöhnlich hohe Temperatur erleiden zu lafen.

Was die zu Anfange von dem Verfaffer erwähnten Vor- 


\section{[ 55: ]}

züge deg Heberbarometers vor dem Gefafsbarometer betrifft, fo find fio bei weitsm nicht fo entflieden, wie fie hler ausgefprochen wurden. Ein jede hat feine befonderen Vorzïge, und wenn es auch bei dem erfteren vermoge feiner einfacheren Einrichtung leichter möglich ift, eine fcharfe Meffung der Queckfilberfäule zu erhalten, fo ift damit noch gar nicht gefagt, dafs beim Gefüfsbarometer nicht auch Genauigkeit zu erreichen wäre. Die Belitzer der Fortin'fchen Gefälsbarometer z. B. wïden gewifs fclwerlich der Behauptung des Verfalfers beitreted wollew. Für Höhenmefrungen auf Reifen, bei wel. chen nie eine grofse Genauigkeit möglich ill, hat das Gefälsbarometer die grofsen Vorzïge, dafs es fich viel ficherer vertlkal ftellt, dafs es wegen foines mehr nach unten vertheilten Gewiclites nicht fo rom Winde bewegt wird, wie das Heberbarometer, und dafs es in der Regel leichter zu transportiren ift, wie jenes. Eben fo ist es für längere Beobachtungen an oinem und demfelben Orto dariu dem Heberbarometer voraus, dafs fein mit der atmofphärifchen Luft in Berührung ftehendes Niveau geringen oder faft unmerklichen Oscillationen unterworfen ift, womit zugleich die Mögliclikeit vermindert wird, dals mit der Zeit Luft in den läugern Sclienkel trete. Auch baben bei gleicher Freite der. Röhren dlo kleinen Unebenheiten und fonftige zufällige Befchaffenheiten der inaeren Flăche des Glafes, beim Heberbarometer weit gröfseren Einflufs auf den Stand der Queckfilberfaule, als beim Gefassbarometer. Es if eine ganz gewühnliche Erfcheinung bei dem erfern, dafs man to viele verfchiedene Ablefungen bekommt, als man, nach vorhergegangener Erfchütterung des Inftrumentes, Einftellungen gemacht hat. Die Vorzüge des Heberbarometers, ein ron den Wirkungen der Capillarität befreites Refultat zu geben, gehören nur denen mit fehr weiter ( 5 bis 6 Linien ftarker) Glasröbre an. Bei Rühren von gewöhnlichem Durchmefler ift im kurzen Schenkel, vermöge der anhaftenden Luft und Feuchtigkeit, die Queckfilberfläche fteis convexer, als in dem langen Schenkel, und zwar in Abhängigkeit von der jedesmaligen hygrometrifchen Befchaffenheit der Luft, fo dafs der Unterfchied nicht einmal ganz conftant bleibt. Zur vollen Genauigkeit hat man 


\section{[ 353 ]}

hier für den kurzen Schenkel eine verånderliche, von der Chorde und der jedesmaligen Sagitte des Bogens abhăngende Correction anzubringen, wozu man fich der von Eckardt und Schleiermacher berechneten Tafeln bedienen kann. Dle in der Connaissance de tems pour 1812 gegebene Tafel für die Capillarität fititzt fich darauf, dafs an dem auf der Sternwarte zu Paris befindlichen Barometer die Wölbungen in beiden Schenkeln gleich waren; eben daffelbe gilt von der Tafel, die Bouvard fpäterhin berechnet hat (Annal. de Chim. et Phys. XXII. 331). Dafs diefe Tafel aber nur für die gemachten Vorausfetzungen gilt, und dafs man z. B. fehr irren würde, wenn man an einem Gefüfsbarometer, deffen oberes Niveau eine Ebene bildet, noch eine dem Durchmeffer entfprechende Correction addirt, ift aus der Canillaritätslehre einieuchtend. Delcrors hat in der Biblioth, univers. VIII. Io diefes allgemeiner zur Sprache gebracht, und dafelbft die oben erwähnte Ta$\mathrm{fel}$ von doppeltem Eingange mitgetheilt, welche auch $\mathrm{Hr}$. Hofr. Murke feinem Lehrbuche und dem Artikel: Barometer, in der neuen Bearbeitung des Gehlerfchen Wörterbuchs beigefügt hat. Man kann dafelbft das Ausfuhrlichere nachfehen. Uebrigens murs noch bemerkt werden, dafs, wenn man niclit die Capillaritäts - Wirkung auf obige Art berückfichtigt und die Queckfilberfäule nicht mit äufserfter Sorgfalt mifst (z. B. die Scale nicht ebeutalls ihr befonderes Thermometer befitzt), auch kein erheblicher Nachtheil aus der gewöhnlich fchwach konifchen Form der Glasröhren entfpringt, fobald man nur diefe Röhren nicht von zu kleinem Durchmeffer nimmt, und beide Niveaux wirklich mifst, nicht das eine durch Rechnung beftimmen will. Dals der Hr. Verfaffer die Queckfilberfläche von da abmifst, wo fie die Glaswand berührt, und nicht vom höchften Punkte ihrer Wölbung, kann wobl nicht allgemein empfohlen werden; denn nur die mittleren Theile der Säule find es, die fich ungehindert mit dem Drucke der Atmofphäre in Gleichgewicht ftellen können.

Herr Dr. Romershaufen hat die hier befchriebene Einrichtung des Barometers noch dahin abgeändert, dafs er den Stempel im unteren Theile der Glasröhre durch eine gezahnte 


\section{[ 354 ]}

Stange heben und fenken, und mfttelft dlefer einen Zeiger in Bewegung fetzen lafst, der den Stand der Queckfilberfäule auf einer getheilten Kreisfcheibe anglebt. Die Ebene der Schenkel fteht dabel fenkrecht gegen die :ibene des Zifferblattes und man hebt mittelft der unteren Schraube den Stem. pel bei jedesmaliger Beobachtung fo weit, bis das Queckfilber im untern Schenkel mit der feften Linie $n n$ in Niveau liegt. Der längere Schenkel ît dabei ganz von der Faffung bedeckt; will man ihn aber fichtbar laffen, fo ftellt man das obere $\mathrm{Ni}$ veau auf den feften Punkt ein. Die Werthe der Theilungen des Zifferblattes werden durch Vergleich des Standes diefes Barometers mit dem eines guten Heberbarometers gefunden. Der Verfaffer nent diefes Inftrument ein Zeigerbarometer. Fig. 6 zeigt die wefentlichen Theile deffelben, und macht eine ausführlicho Befchreibung unnöthig, da ïberdiefs diefe Einrichtung mehr auf Beluftigung als auf wirklichen Nutzen bo. rechnet ift. Dafs die Queckfilbermenge in diefem Inftrumente ftets diefelbe bleiben mufs, if eine Eigenfchatt, die daffelbe nicht zu feinem Vortheil auszeichnet; auch weifs ich nicht ob man diefes Zeigerbarometer als eine Verbefferung des Hookefchen Radbarameters zu betrachten hat, denn von Genanigkeit und Zuverläffigkeit in den Angaben, kann ftrenge genommen bei beiden nicht die Rede feyn, und als Wetterglas, empfiehlt fich das letztere dem Beobachter dadurch, dars es der Mühe des Einfellens iberhebt. Ohne im Geringften den verdienftichen Bemühungen des Hrn. Verfaffers Eintrag thun zu wollen, kann ich mich doch der Bemerkung nicht enthalten, dars die Barometer, wie fie in netuerer Zeit aus den befie. ren Werkfätten Deutfchlands und Frankreichs hervorgegangen find, den Zwecken der Meteorologie vollkommen Genüge leiften; und das Heil der Wiflenfchaft nur davon abhingt, diefe Inftrumente unbefchadet ihrer Güte minder koftbar zu machen, fie allgemeiner $z u$ verbreiten, und nach einem geordneten Plane anhaltend zu beobachten. $P$. 